Cite this: RSC Adv., 2014, 4, 18136

\title{
High internal phase emulsion gels (HIPE-gels) prepared using food-grade components $\dagger$
}

\author{
Ashok R. Patel, ${ }^{\text {*a }}$ Ysamar Rodriguez, $^{\mathrm{b}}$ Ans Lesaffer ${ }^{\mathrm{b}}$ and Koen Dewettinck ${ }^{\mathrm{a}}$
}

Received 11th March 2014

Accepted 2nd April 2014

DOI: $10.1039 / c 4 r a 02119 c$

www.rsc.org/advances

We report a new approach of using dispersed water phase gelation as a mode to create oil continuous emulsion gels. The low temperature gelation property of synergistic hydrocolloid combinations was exploited to develop elastic soft solids using only food-grade components.

Many of the regularly consumed food products are emulsionbased soft solids that display gel-like behaviour (structured emulsions).$^{1,2}$ These viscoelastic products can be broadly categorized into three main classes including: (i) water continuous emulsions structured using proteins and/or polysaccharides that act as emulsifiers as well as thickening or gelling agents (e.g. cheese, yogurt, milk-based deserts etc.); (ii) highly concentrated water continuous emulsions where the rheological structuring comes from the close crowding of dispersed oil droplets at high packing fractions (such as mayonnaise, sauce etc.) and (iii) oil continuous emulsions structured using network of fine solid fat crystals (e.g. margarine, butter and spreads)..$^{3-5}$

While a lot of research has been conducted on studying and identifying ways to structure the water continuous emulsions, the exploration of alternate ways to structure oil continuous emulsions has been lagging far behind with most commercial products still relying on the use of solid fats for structure creation. ${ }^{5}$ In wake of the recent reports on health concerns relating to the consumption of saturated and trans-fats, some research has emerged in last few years where structured oil continuous emulsions have been prepared in absence of solid fats via oleogelation. ${ }^{6-11}$ However, the oleogelators used in these studies are either not suitable for food-grade applications (12-hydroxy stearic acid and waxes) $)^{12-16}$ or are rather expensive

${ }^{a}$ Vandemoortele Centre 'Lipid Science \& Technology', Lab. of Food Tech. \& Engg., Faculty of Bioscience Engg., Ghent University, Belgium. E-mail: Patel.Ashok@Ugent. be; Fax: +32 9264 6218; Tel: +329264 6209

${ }^{b}$ Vandemoortele $R \&$ D Centre, Prins Albertlaan 79, 8870 Izegem, Belgium

$\dagger$ Electronic supplementary information (ESI) available. See DOI: $10.1039 / \mathrm{c} 4 \mathrm{ra0} 2119 \mathrm{c}$ (phytosterols and oryzanol) to be considered for use in routine food products. ${ }^{17}$ There are some interesting non-food related research results where high internal phase emulsion (HIPE) organogels were achieved through the use of low molecular weight gelators and functionalized polymers (both of which requires a significant synthetic efforts and are non-food grade). ${ }^{18,19}$

In this paper, we report for the very first time a new approach to create structured soft solids based on gelation of oil continuous HIPE. The gelling of the emulsion was achieved through the use of food-grade hydrocolloids dispersed in the internal phase that constituted major volume of the emulsion $\left(\varphi_{\text {water }}>\right.$ close packing volume). Galactomannans (natural polymers made up of galactose and mannose subunits) are known to show synergistic interactions with other food-grade hydrocolloids like carrageenan (CAR) and xanthan gum (XAN). In particular, locust bean gum (LBG), a galactomannan with a higher mannose : galactose ratio $(\sim 4: 1)$ displays a strong interaction with other non-gelling hydrocolloids leading to the formation of gel at a low total polymer concentration..$^{20}$ In the current work, the synergistic gelling property of these hydrocolloid combinations was exploited to generate oil continuous HIPE gel by first finely dispersing hydrocolloid solution in oil continuous medium through emulsification at high temperature $\left(>70^{\circ} \mathrm{C}\right)$ followed by cooling to room temperature to trigger water droplet gelation (Fig. 1a).

Due to the high phase volume, the gelled droplets provided a structural framework that held the emulsion together, resulting in a self-standing elastic gel-like structure. The effect of phase volume on the structuring is evident from Fig. 1b. At lower phase volumes $\left(\varphi_{\text {water }}=0.25\right.$ ), the formed emulsion was fluid in nature and did not show any gelation. At higher phase volumes $\left(\varphi_{\text {water }}=0.5\right.$ and 0.75 ), gelation of both emulsions was evident. However, emulsion with $\varphi_{\text {water }}=0.5$ showed physical instability overnight, leading to a distinct separation into a gel phase and a fluid phase (Fig. 2a). The optical microscopy images shown in Fig. $2 c \& d$ indicate that the emulsion in both the gel and separated fluid phase was stable with higher droplet population 

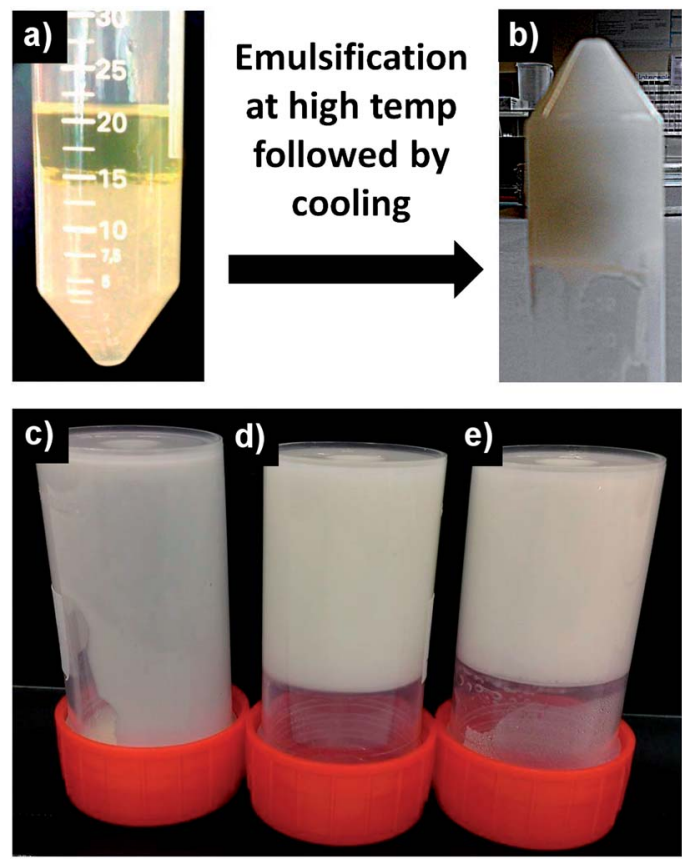

Fig. 1 (a \& b) The preparation process involved a high temperature $\left(>70{ }^{\circ} \mathrm{C}\right.$ ) emulsification of a mixture of oil and water (containing hydrocolloids) in presence of low HLB (hydrophilic lipophilic balance) emulsifier PGPR (polyglycerol polyricinoleate) followed by cooling to room temperature leading to the immediate formation of an oil continuous emulsion gel; ( $c, d, \&$ e) emulsions prepared at internal phase volume of $0.25,0.5$ and 0.75 respectively.

in close packing constituting the gelled phase and a dilute emulsion making up the separated fluid phase. In both cases, the emulsion did not show any coalescence. The emulsion with $\varphi_{\text {water }}=0.75$ on the other hand, gave an elastic self-standing gel

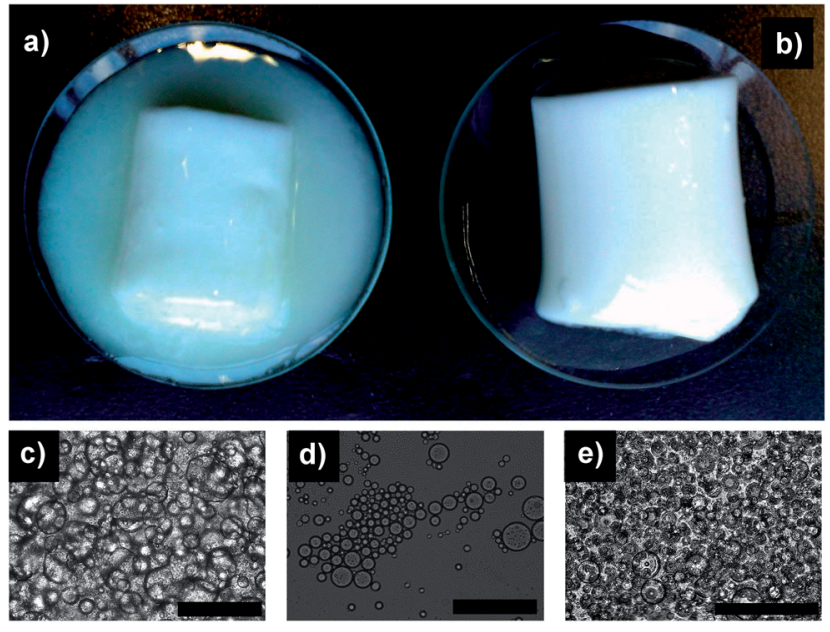

Fig. 2 (a \& b) Photographs of emulsion gels prepared at internal phase volumes ( $\varphi_{\text {water }}$ ) of 0.5 and 0.75 respectively; (c $\&$ d) optical microscopy image (scale bars $=25 \mu \mathrm{m}$ ) of separated gel and fluid phases respectively of emulsion prepared at $\varphi_{\text {water }}=0.5$. Notice the microstructure difference of the two phase; (e) optical microscopy image (scale bar $=25 \mu \mathrm{m}$ ) of HIPE gel prepared at $\varphi_{\text {water }}=0.75$.
(Fig. 2b) which was stable for over 3 months without showing any separation or droplet size increase. The tightly packed, gelled water droplets were seen under the microscope (Fig. 2e) suggesting that the close packing of gelled emulsion droplets is necessary to build a framework to support the 3D gel structure that physically traps oil phase in the inter-droplet sites lined by crowded interfaces.

Initial emulsification trials without hydrocolloids were carried out using commonly used food emulsifiers - monoglycerides (consisting of a fatty acid linked to glycerol backbone). ${ }^{21,22}$ However, due to their limited surface activity, the emulsions formed using monoglycerides even at concentrations as high as 5\% wt were unstable and showed phase separation on cooling (Fig. S1a $\dagger$ ). On the other hand, stable HIPE at $\varphi_{\text {water }}=$ 0.75 (with a mean droplet radius of $4.39 \mu \mathrm{m}$ ) was obtained by using polyglycerol polyricinoleate (PGPR) at a low concentration of $0.4 \%$ wt (Fig. S1b $\dagger$ ). Unlike monoglycerides, PGPRs are polymeric surfactants (fatty acid esters of polyglycerol) ${ }^{23}$ with strong surface activity ${ }^{22}$ used for specialized food formulations like chocolate, low fat spreads and bakery products. ${ }^{24}$ The HIPE gels were formed by incorporating hydrocolloids (a combination of LBG:CAR and LBG:XAN) in the water phase. Though the hydrocolloids used are non-surface active, the HIPE gels had lower average droplet sizes (3.5 and $2.76 \mu \mathrm{m}$ for LBG:CAR and LBG:XAN respectively) then HIPE emulsion (Fig. S2 $\dagger$ ). The probable reason for this decrease in the water droplet size could be due to the increase in the viscosity of the internal phase due to the incorporation of hydrocolloids. The decrease in the droplet size with the increase in the viscosity of dispersed phase could be explained by the fact that under the shear flow, the high viscosity droplets can resist disruption longer and thus undergo higher deformation before breaking down into smaller droplets. ${ }^{22,25}$ LBG:CAR and LBG:XAN are well-studied synergistic combinations used as formulation aid in various food products. ${ }^{\mathbf{2 6 - 2 8}}$ The exact mechanism of these synergistic interactions are still unclear but they are utilized to obtain gels in combination with non-gelling hydrocolloids. ${ }^{\mathbf{2 8} 29}$ The gels formed by these hydrocolloid combinations are low temperature setting and thermo reversible. ${ }^{\mathbf{2 0 , 3 0}}$ The thermo reversible property of these hydrocolloid combinations was used to form HIPE gels by emulsifying the melted gel with oil at high temperature followed by cooling down to the room temperature in order to trigger gel formation of the internal phase.

The microstructure of HIPE gel was studied using a range of microscopy (optical, confocal and cryo-SEM) techniques (Fig. 3). Visualization of emulsion under confocal microscope confirmed the oil-continuous nature of emulsion with dispersed gelled water droplets closely packed together (Fig. 3b $\& \mathrm{c})$. The deformation of droplet shapes indicates close packing structure of the emulsion which forms the main framework that supports the $3 \mathrm{D}$ gel structure. The gelled water droplets are further seen in the cryo-SEM images of freeze-fractured HIPE gel (Fig. 3c \& d). The HIPE gels were stable over 3 months of storage as indicated by an absence of "oiling-off", no coalescence and uniformity of average droplet size (Fig. S3†).

Food emulsion gels have interesting viscoelastic properties which are usually evaluated by studying their large deformation 

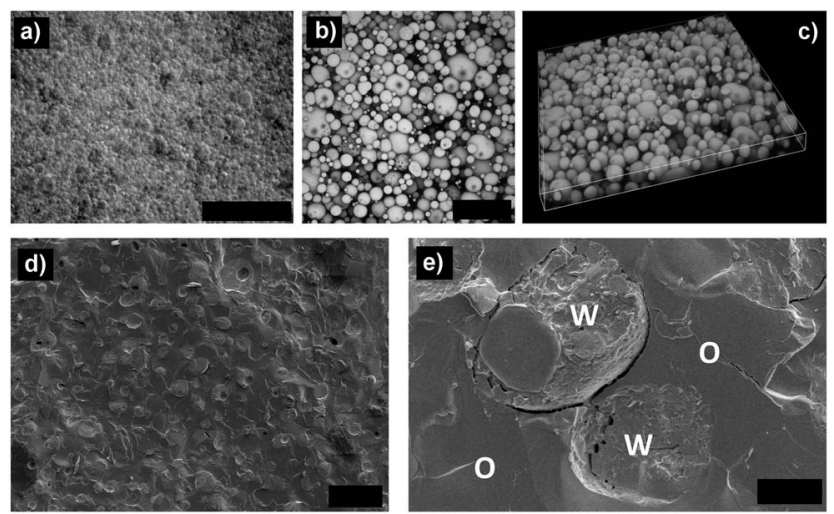

Fig. 3 (a \& b) Optical and confocal microscopy images of HIPE gel prepared at $\varphi_{\text {water }}=0.75$ (scale bars $=100 \& 20 \mu \mathrm{m}$ respectively); (c) $3 \mathrm{D}$ volume view created using stacked images obtained from confocal microscopy (sample depth = $15 \mu \mathrm{m}$ ); (d) cryo-SEM image of freeze fractured HIPE gel showing gelled droplets in an oil continuous phase (scale bar $=10 \mu \mathrm{m}$ ); (e) the fractured gelled water droplet (scale bar $=2$ $\mu \mathrm{m})$, water (W) and oil (O) phase are marked for better clarity. The HIPE gel was prepared using $0.4 \%$ wt of emulsifier (PGPR) and a total polymer concentration of $1 \%$ wt (containing LBG:CAR in $75: 25$ ratio).

fracture properties and yielding behaviour..$^{1,31-33}$ The HIPE gels were subjected to uni-axial compression testing to gain more information about the structural arrangement of these systems. Both the total polymer concentration as well as polymer proportions in the combinations had an effect on the fracture properties of HIPE gels. The increase in the polymer concentration resulted in a decrease of fracture strain as indicated by a decrease in the displacement values $\left(l^{*}\right)$ at fracture point or point of permanent deformation (Fig. $4 \&$ Table S1†). The modulus of elasticity (Young's modulus, $E_{\mathrm{y}}$ ) is a measure of the stiffness (rigidity) of a sample or in other words, the extent to which a sample resists deformation to an applied force. ${ }^{33,34}$ The values of $E_{\mathrm{y}}$ is calculated from the slope of the linear portion of load-displacement curve where the load is below elastic limit and the sample still obeys Hooke's law (i.e. load is proportional to extension of sample). ${ }^{34}$ On the other hand, critical force $\left(F^{*}\right)$ is a measure of the strength of a sample i.e. the ability to withstand an applied stress without failure..$^{35} \mathrm{In}$ terms of gels, a higher $E_{\mathrm{y}}$ indicates a stiffer gel that can be stretched only to a
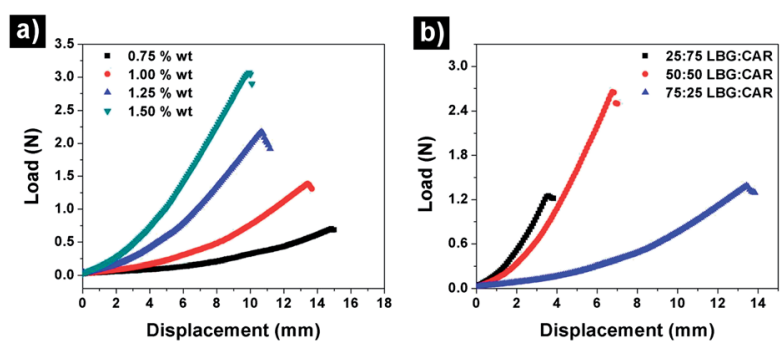

Fig. 4 Load-displacement curves for HIPE gels made with LBG:CAR at (a) different total polymer concentrations while keeping the LBG:CAR proportion at $75: 25$ and (b) different ratios of LBG:CAR at a total polymer concentration of $1 \%$ wt. PGPR was used as an emulsifier in this emulsions at concentration of $0.4 \% \mathrm{wt}$. certain extent before it breaks and a higher fracture force indicates the higher gel strength or gel hardness. ${ }^{36,37}$ A desirable food emulsion gel needs to have a reasonable strength (intermediate to high $F^{*}$ ) but at the same time a lower $E_{\mathrm{y}}$ and a higher fracture strain (higher displacement at fracture point, $l^{*}$ ) so that it is stretchable (and consequently spreadable) over a larger surface before it undergoes plastic deformation. Understandably, the increase in the total polymer concentration led to an increase in the values of $F^{*}$ and $E_{\mathrm{y}}$ while showing a decrease in the values of $l^{*}$. The HIPE gel prepared at a total polymer concentration of $0.75 \%$ wt showed lowest $E_{\mathrm{y}}\left(4.62 \mathrm{~N} \mathrm{~m}^{-2}\right)$ but was very weak as indicated from a very low value of $F^{*}(0.69 \mathrm{~N})$. On the other hand, samples made at $1.25 \%$ and $1.5 \%$ wt polymer concentration showed good gel strength $(2.17$ and $3.07 \mathrm{~N}$ respectively) but were very rigid $\left(E_{\mathrm{y}}=15.78\right.$ and $23.23 \mathrm{~N} \mathrm{~m}^{-2}$ for 1.25 and $1.5 \%$ wt respectively) and showed a lower spreadability $\left(l^{*}=10.67\right.$ and $9.92 \mathrm{~mm}$ for 1.25 and $1.5 \%$ wt respectively). A total polymer concentration of $1 \% \mathrm{wt}$ showed a reasonable gel strength $\left(F^{*}>1 \mathrm{~N}\right)$ as well as a high fracture strain $\left(l^{*}=\right.$ $13.42 \mathrm{~mm}$ ) and hence, it was selected for further fracture property evaluation by varying the proportion of hydrocolloids in the emulsion gels (Fig. 4b). As usually seen in LBG:CAR gels, ${ }^{38}$ the increase in the LBG proportion led to an increased elasticity of emulsion gels as indicated from the increase in the fracture strain $\left(l^{*}=3.59 \mathrm{~mm}\right.$ for LBG:CAR, $25: 75$ to $l^{*}=13.42 \mathrm{~mm}$ for LBG:CAR, $75: 25)$. On the other hand, a stiff gel with high gel strength $\left(E_{\mathrm{y}}=19.18 \mathrm{~N} \mathrm{~m}^{-2}\right.$ and $\left.F^{*}=2.65 \mathrm{~N}\right)$ was obtained at LBG:CAR proportion of $50: 50$. The increased gel strength at a $50: 50$ ratio can be explained based on the synergistic interactions between carrageenan and LBG which are strongest at proportions of LBG:CAR between $60: 40$ to $40: 60,{ }^{39}$ the interactions are due to the involvement of the smooth un-branched segments of D-mannose backbone in H-bonding. ${ }^{26,40}$ In our study, LBG:CAR ratio of $75: 25$ was selected as a balance between required elasticity (higher $l^{*}$ ) and intermediate stiffness (lower $E_{\mathrm{y}}$ ).

The HIPE gels prepared using hydrocolloid combinations of LBG:CAR and LBG:XAN at a total polymer concentration of $1 \%$ wt (and LBG proportion of $75 \%$ in the hydrocolloid combinations) were further compared using relaxation test and 2-cycle compression tests or texture profile analysis (TPA). The comparative relaxation curves (Fig. 5a) confirms the viscoelastic behaviour of these gels where a prominent stress relaxation was observed under a constant strain. In other words, the stress required to maintain the constant strain decreased with the increase in time. ${ }^{41}$ Depending on the type of gels, such behaviour is explained via two possible mechanisms: (a) diffusiondriven relaxation due to the transport of water in and out of the gel network (seen in chemical gels) or (b) network rearrangement based weakening of physical linkages (physical gels)..$^{42}$ In our case, since there was no fluid release from the gels at the end of these tests, the stress relaxation can be speculated to be due to the weakening of the network of closely packed gelled water droplets resulting in the progressive loss of energy storage capability of the structural framework. Additionally, the deformation of gelled water droplets will also have a significant role to play. Even though we are working at a high dispersed phase 

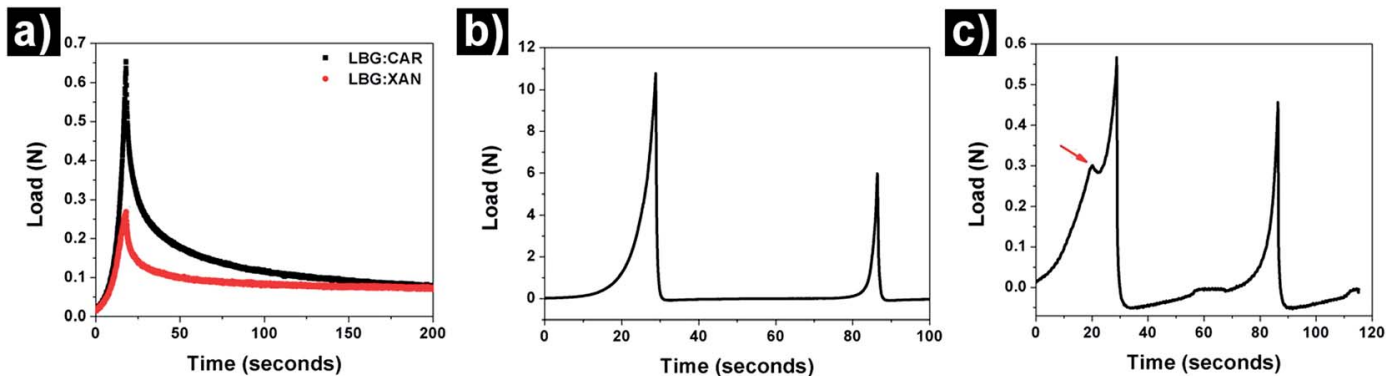

Fig. 5 (a) Comparative curves obtained from relaxation tests done on HIPE gels made using LBG:CAR and LBG:XAN; (b \& c) TPA curves for HIPE gels made using LBG:CAR and LBG:XAN respectively.

Table 1 Textural parameters of HIPE gels made using LBG:CAR and LBG:XAN

\begin{tabular}{|c|c|c|}
\hline Parameters & LBG:CAR & LBG:XAN \\
\hline Firmness (N) & $10.79 \pm 0.61$ & $0.57 \pm 0.01$ \\
\hline Cohesiveness $^{a}$ & $0.19 \pm 0.02$ & $0.23 \pm 0.01$ \\
\hline Springiness index ${ }^{a}$ & $0.48 \pm 0.03$ & $0.44 \pm 0.03$ \\
\hline Adhesiveness $\left(\mathrm{N} \mathrm{m}^{-2}\right)$ & - & $1.7 \times 10^{-3}$ \\
\hline Fracturability (N) & - & $0.29 \pm 0.001$ \\
\hline Resilience $^{a}$ & $0.07 \pm 0.003$ & $0.04 \pm 0.002$ \\
\hline Chewiness $\left(\mathrm{N} \mathrm{m}^{-2}\right)$ & $0.98 \pm 0.06$ & $0.06 \pm 0.01$ \\
\hline
\end{tabular}

volume fractions, the microscopy evaluation show that the droplets are still spherical and haven't undergone a maximum deformation (polygonal shape). Thus, under strain which is lower than the fracture strain, the spherical droplets may undergo further deformation resulting in the restructuring of the network and contributing to the stress dissipation. It can also been seen that the peak load for HIPE gel prepared using LBG:CAR was much higher than LBG:XAN indicating a clear difference in the strength of these HIPE gels. The difference in the properties of these gels were further quantified using parameters (Table 1) obtained from TPA curves (Fig. 5b \& c). The HIPE gel prepared using LBG:XAN had a much lower firmness (0.57 $\mathrm{N}$ as compared to $10.79 \mathrm{~N}$ for LBG:CAR) and showed a prominent brittleness (fracturability $=0.29 \mathrm{~N}$ ) as indicated by red arrow in Fig. 5c. The comparatively higher elasticity of LBG:CAR can also be seen from the higher value of chewiness (energy required for breakdown of the gel over consecutive cycles). Moreover, LBG:XAN gel was sticky in nature as indicated by an adhesiveness value of $1.7 \times 10^{-3} \mathrm{~N} \mathrm{~m}^{-2}$ (adhesiveness is a measure of negative work done on the probe during its upward movement) ${ }^{43}$ Hence, it is clear from these results that there was a distinct difference in the large deformation fracture properties of HIPE gels prepared by combining LBG with two different hydrocolloids (CAR and XAN).

\section{Conclusions}

In conclusion, we successfully demonstrated a facile approach of making oil continuous HIPE gels using only common food- grade components (hydrocolloids and a food emulsifier). To develop such systems, two important considerations need to be taken in to account: first is the creation of a stable oil continuous HIPE followed by selecting appropriate hydrocolloids for gelling the internal water droplet phase. The hydrocolloids to be used for these systems should show a thermoreversible, low temperature gelation. A combination of hydrocolloids is more appropriate as it gives us wider options to influence the properties of formed gels based on the polymer proportions. It is also necessary to use hydrocolloids that are non-surface active in order to prevent the inversion of the systems into water continuous gel.

We believe that the possibility of structuring oil continuous gel systems without the use of any solid fats could be of significant importance to food product development especially for lowering of fat levels.

\section{Acknowledgements}

This research is supported by Marie Curie Career Integration Grant within the $7^{\text {th }}$ European Community Framework Programme.

\section{Notes and references}

1 E. Dickinson, Food Hydrocolloids, 2012, 28, 224-241.

2 V. B. Tolstoguzov and E. E. Braudo, J. Texture Stud., 1983, 14, 183-212.

3 M. M. Robins and P. J. Wilde, in Encyclopedia of Food Sciences and Nutrition, ed. B. Caballero, Academic Press, Oxford, 2nd edn, 2003, pp. 1517-1524.

4 E. Dickinson, Curr. Opin. Colloid Interface Sci., 2010, 15, 40-49.

5 A. Bot, E. Flöter, H. P. Karbstein-Schuchmann and H. S. Ribeiro, in Product Design and Engineering, Wiley-VCH Verlag GmbH \& Co. KGaA, 2013, pp. 315-343.

6 A. Bot and W. M. Agterof, J. Am. Oil Chem. Soc., 2006, 83, 513521.

7 A. Bot and E. Floter, in Edible Oleogels: Structure and Health Implications, ed. A. G. Marangoni and N. Garti, AOCS Press, Urbana, IL, USA, 2011, pp. 49-79.

8 L. S. K. Dassanayake, D. R. Kodali and S. Ueno, Curr. Opin. Colloid Interface Sci., 2011, 16, 432-439. 
9 T. Dey, D. A. Kim and A. G. Marangoni, in Edible Oleogels: Structure and Health Implications, ed. A. G. Marangoni and N. Garti, AOCS Press, Urbana, Illinois, 2011, pp. 295312.

10 A. Marangoni, J. Am. Oil Chem. Soc., 2012, 89, 749-780.

11 A. G. Marangoni, N. Acevedo, F. Maleky, E. Co, F. Peyronel, G. Mazzanti, B. Quinn and D. Pink, Soft Matter, 2012, 8, 1275-1300.

12 Edible oleogels: Structure and health implications, ed. A. G. Marangoni and N. Garti, AOCS Press, Urbana, Illinois, 2011.

13 A. R. Patel, D. Schatteman, W. H. De Vos, A. Lesaffer and K. Dewettinck, J. Colloid Interface Sci., 2013, 411, 114-121.

14 A. R. Patel, D. Schatteman, W. H. D. Vos and K. Dewettinck, RSC Adv., 2013, 3, 5324-5327.

15 J. Toro-Vazquez, J. Morales-Rueda, V. A. Mallia and R. Weiss, Food Biophys., 2010, 5, 193-202.

16 A. R. Patel, P. S. Rajarethinem, A. Gredowska, O. Turhan, A. Lesaffer, W. H. De Vos, D. Van de Walle and K. Dewettinck, Food Funct., 2014, 5, 645-652.

17 H. Sawalha, R. den Adel, P. Venema, A. Bot, E. Flöter and E. van der Linden, J. Agric. Food Chem., 2012, 60, 3462-3470.

18 Y. Chen, N. Ballard, F. Gayet and S. A. F. Bon, Chem. Commun., 2012, 48, 1117-1119.

19 T. Zhang and Q. Guo, Chem. Commun., 2013, 49, 1180311805.

20 Functional Properties of Food Macromolecules, ed. S. E. Hill, D. A. Ledward and J. R. Mitchell, Aspen Publishers Inc., Maryland, USA, 1998.

21 A. Sein, J. A. Verheij and W. G. M. Agterof, J. Colloid Interface Sci., 2002, 249, 412-422.

22 Encylcopedic Handbook of Emulsion Technology, ed. J. Sjoblom, Marcel Dekker Inc., New York, USA, 2001.

23 N. Garti, A. Aserin and B. Zaidman, J. Am. Oil Chem. Soc., 1981, 58, 878-883.

24 Food Emulsifiers and Their Applications, ed. G. N. Hasenhuetll and R. W. Hartel, Chapman \& Hall, New York, USA, 1997.

25 S. R. Derkach, International Review of Chemical Engineering, 2010, 2, 465-472.
26 M. Tako, Z.-Q. Qi, E. Yoza and S. Toyama, Food Res. Int., 1998, 31, 543-548.

27 R. O. Mannion, C. D. Melia, B. Launay, G. Cuvelier, S. E. Hill, S. E. Harding and J. R. Mitchell, Carbohydr. Polym., 1992, 19, 91-97.

28 Food Polysaccharides and Their Applications, ed. A. M. Stephan and G. O. Phillips, CRC Press, FL, USA, 2006.

29 D. F. Zhan, M. J. Ridout, G. J. Brownsey and V. J. Morris, Carbohydr. Polym., 1993, 21, 53-58.

30 M. Tako, T. Teruya, Y. Tamaki and K. Ohkawa, Colloid Polym. Sci., 2010, 288, 1161-1166.

31 G. Sala, R. A. de Wijk, F. van de Velde and G. A. van Aken, Food Hydrocolloids, 2008, 22, 353-363.

32 G. Sala, F. van de Velde, M. A. Cohen Stuart and G. A. van Aken, Food Hydrocolloids, 2007, 21, 977-985.

33 Texture in Food, ed. D. Kilcast, Woodhead Publishing Ltd \& CRC Press, Cambridge, UK, 2004.

34 Food Texture: Instrumental and Sensory Measurement, ed. H. R. Moskowitz, Marcel Dekker Inc., New York, USA, 1987.

35 D. D. Hamann, J. Zhang, C. R. Daubert, E. A. Foegeding and K. C. Diehl, J. Texture Stud., 2006, 37, 620-639.

36 G. Sala, G. A. Van Aken, M. A. C. Stuart and F. Van De Velde, J. Texture Stud., 2007, 38, 511-535.

37 G. Sala, T. van Vliet, M. A. Cohen Stuart, G. A. v. Aken and F. van de Velde, Food Hydrocolloids, 2009, 23, 1381-1393.

38 D. E. Dunstan, Y. Chen, M. L. Liao, R. Salvatore, D. V. Boger and M. Prica, Food Hydrocolloids, 2001, 15, 475-484.

39 M. Srivastava and V. P. Kapoor, Chem. Biodiversity, 2005, 2, 295-317.

40 A. C. Pinheiro, A. I. Bourbon, C. Rocha, C. Ribeiro, J. M. Maia, M. P. Gonçalves, J. A. Teixeira and A. A. Vicente, Carbohydr. Polym., 2011, 83, 392-399.

41 M. Mancini, M. Moresi and R. Rancini, J. Texture Stud., 1999, 30, 639-657.

42 G. Gentile, F. Greco and D. Larobina, Eur. Polym. J., 2013, 49, 3929-3936.

43 Food Texture and Viscosity: Concept and Measurement, ed. M. C. Bourne, Academic Press, California, USA, 2002. 